Article

\title{
Cylindrical Dielectric Resonator Antenna-Based Sensors for Liquid Chemical Detection
}

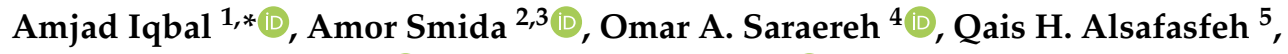 \\ Nazih Khaddaj Mallat ${ }^{6}$ and Byung Moo Lee ${ }^{7, *(D)}$ \\ 1 Centre for Wireless Technology (CWT), Faculty of Engineering, Multimedia University, \\ Cyberjaya 63100, Malaysia \\ 2 Department of Medical Equipment Technology, College of Applied Medical Sciences, Majmaah University, \\ AlMajmaah 11952, Saudi Arabia; a.smida@mu.edu.sa \\ 3 Unit of Research in High Frequency Electronic Circuits and Systems, Faculty of Mathematical, \\ Physical and Natural Sciences of Tunis, Tunis El Manar University, Tunis 2092, Tunisia \\ 4 Department of Electrical Engineering, Hashemite University, Zarqa 13115, Jordan; eloas2@hu.edu.jo \\ 5 Department of Electrical Power and Mechatronics Engineering, Tafila Technical University, \\ Tafila 11183, Jordan; qsafasfeh@ttu.edu.jo \\ 6 College of Engineering, Al Ain University of Science and Technology, Al Ain 64141, UAE; \\ nazih.mallat@aau.ac.ae \\ 7 School of Intelligent Mechatronics Engineering, Sejong University, Seoul 05006, Korea \\ * Correspondence: aiqbal@ieee.org (A.I.); blee@sejong.ac.kr (B.M.L.); Tel.: +92-344-804-5575 (A.I.)
}

Received: 23 February 2019; Accepted: 5 March 2019; Published: 8 March 2019

check for updates

\begin{abstract}
A compact, cylindrical dielectric resonator antenna (CDRA), using radio frequency signals to identify different liquids is proposed in this paper. The proposed CDRA sensor is excited by a rectangular slot through a 3-mm-wide microstrip line. The rectangular slot has been used to excite the CDRA for $H E M_{11}$ mode at 5.25 GHz. Circuit model values (capacitance, inductance, resistance and transformer ratios) of the proposed CDRA are derived to show the true behaviour of the system. The proposed CDRA acts as a sensor due to the fact that different liquids have different dielectric permittivities and, hence, will be having different resonance frequencies. Two different types of CDRA sensors are designed and experimentally validated with four different liquids (Isopropyl, ethanol, methanol and water).
\end{abstract}

Keywords: dielectric resonator; chemical sensing; S-parameters; circuit model

\section{Introduction}

Chemical sensors have been utilized for a long time to distinguish the purity/impurity of several liquids/chemicals to improve the course of action of those liquids/chemicals for an extensive variety of modern applications. It is important to store the liquids/chemicals as per the Globally Harmonized System of Classification and labelling of liquids/chemicals. The utilization of nameless liquids/chemicals may lead to unexpected results including harming human organs. Thus, liquids/chemicals ought to be marked with the goal that they are perceived appropriately [1].

Material characterization and sensing have recently gained much more attention due to the fast developing interest in various industries. Each material has a distinctive behaviour, based on the properties and nature of the dielectric material in the presence of the electromagnetic fields. The nature and behaviour of the dielectric material are determined by analyzing properties such as permittivity, permeability and conductivity. Permittivity is a vital characteristic among all the properties, based on which the electrical nature of the material is determined. Various types of sensors and sensor combinations are utilized for chemical sensing and material characterization in different industries; 
however, these methodologies have some disadvantages like high power consumption, large size, complicated design and uneconomical usage [2]. Radiofrequency (RF) and microwave sensing for chemical identification and material characterization is considered one of the best sensing techniques because of its cost-effectiveness, low power consumption and easy fabrication [3-5].

Formerly, the techniques used for looking at bioassays and evaluating fluid quality required enormous volumes of fluids/chemicals to fill the testing tubes for analysis [6-8]. An extensive amount of those fluids remain unused, disposed of and never utilized for exploratory necessities. For eliminating this issue of squandering unused fluidic chemicals, microfluidic courses of action were declared. Investigation of the fluids/chemicals would now be able to be accomplished on completely little volumes, typically at the micro-liter or nano-litre range of the fluids/chemicals. Liquid/chemical sensing technology has gained much more advancement through the development of chip-based compact waveguides [9-11]. A high-quality factor, SIW resonator, having confined energies at the centre, is utilized as a humidity-sensing sensor [8]. A gas detection sensor is designed using a multi-mode (Specifically $T E_{101}$ and $T E_{102}$ ) SIW resonator [12]. A complementary split ring resonator-based sensor for liquid dielectric characterization is reported in [13], in which the material under testing is placed in a normal orientation to the sensor surface.

Several attempts for performance enhancement have been carried out while keeping the sensor as small as possible. Several Substrate Integrated Waveguide (SIW) based sensors were designed for different sensing applications. Two unequal microfluidic channels are placed in highly intensive electric field regions in the SIW cavity for dual chemical detection [14]. Circular SIW cavity is utilized for single chemical detection [15]. Dual mode resonator utilizing a folded microstrip line is utilized for dual chemical sensing [16]. A number of studies have been carried out for ethanol liquid sensing using SIW [17-19]. An inject-printed microstrip patch antenna is used as a sensor for liquid identification in [20].

In this paper, a cylindrical dielectric resonator antenna-based sensor is proposed and experimentally validated for different chemicals (Isopropyl, ethanol, methanol and water). The proposed DRA-based sensors shows a reasonable sensing capability as compared to the existing sensing technologies. Main contributions of the proposed work are given below

- To the best of our knowledge, this is the first ever DRA-based constructed sensor for chemical liquid detection.

- A minimal size, large frequency shift and high sensitivity make it a good candidate to be used for chemical sensing.

\section{Design Methodology}

\subsection{Dielectric Resonator Antenna Characterization}

Figure 1 shows the configuration of the proposed cylindrical dielectric resonator antenna. The radiating element (CDRA) is placed on a $1.6 \mathrm{~mm}$ thicker FR-4 substrate with a relative permittivity of 4.4 and a loss tangent of 0.019 . The width and length of the substrate are chosen as $30 \mathrm{~mm}$. TMM 10i material having a relative permittivity of 9.8 and loss tangent of 0.002 is utilized for designing the CDRA. The CDRA has a height of $9 \mathrm{~mm}$ and a radius of $6.35 \mathrm{~mm}$. To feed the antenna, a $50 \Omega$ microstrip line is used. A rectangular slot below the CDRA is designed to feed the CDRA. The proposed DRA resonates at $5.25 \mathrm{GHz}$ with $10 \mathrm{~dB}$ bandwidth of $340 \mathrm{MHz}(5.08-5.42 \mathrm{GHz}$ ) covering most of the application bands such as Mi-WiFi, WLAN and ISM band. the proposed antenna have high gain of $7.1 \mathrm{dBi}$ and efficiency of $82 \%$. The proposed DRA can be used in applications such as Mi-WiFi, WLAN and ISM band because of its high quality factor, good impedance matching, high gain and efficiency. Also, the compact structure of the antenna makes it a suitable choice for system-in-package (SIP) applications. 


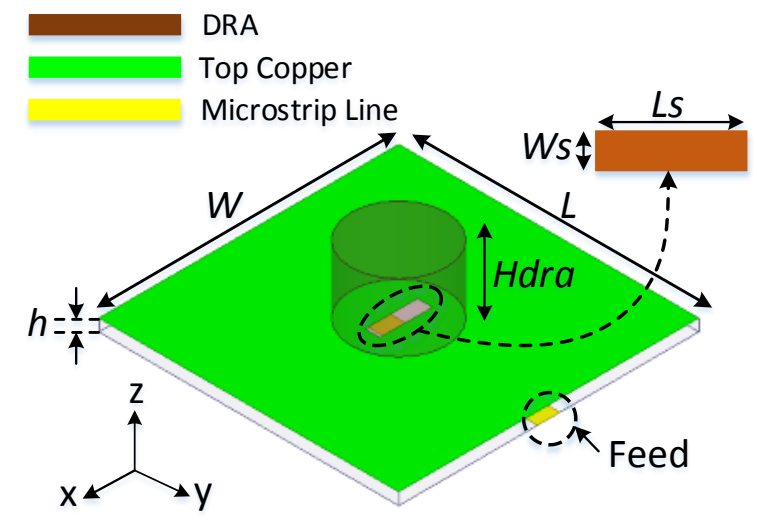

Figure 1. Proposed cylindrical dielectric resonator antenna to be used as a sensor $(L s=7 \mathrm{~mm}, W s=2 \mathrm{~mm}$, Hdra $=9 \mathrm{~mm}, h=1.6 \mathrm{~mm}$ ).

The resonance frequency of a rectangular-shaped slot for fundamental $\left(T M_{10}\right)$ mode can be calculated as follows [21].

$$
f_{r}=\frac{c}{2 L_{s}} \sqrt{\frac{2}{1+\epsilon_{s}}}
$$

where $c$ shows the speed of light and $\epsilon_{s}$ represents permittivity of the substrate which is 4.4. From (1), the resonance frequency of a rectangular shaped slot for fundamental $\left(T M_{10}\right)$ mode is found to be $5.1 \mathrm{GHz}$.

In the same way, the resonance frequency of $H E M_{11}$ mode in CDRA can be calculated as follow [22].

$$
f_{r}=\frac{6.321 c}{\pi D \sqrt{\epsilon_{r, e f f}+2}}\left[0.27+0.36\left(\frac{2 r}{4 H_{e f f}}\right)+0.02\left(\frac{2 r}{4 H_{e f f}}\right)^{2}\right]
$$

where $r$ represents the radius of the CDRA, $H_{e f f}$ represents the effective height of the resonator. $H_{e f f}$ is the sum of the height of the CDRA and substrate thickness ( $h+h d r a) . \epsilon_{r, e f f}$ is the effective permittivity of the proposed CDRA and can be calculated as [22].

$$
\epsilon_{r, e f f}=\frac{H_{e f f}}{\frac{H}{\epsilon_{r, C D R A}}+\frac{H}{\epsilon_{r, s u b}}}
$$

\section{Excitation Mechanism and Equivalent Circuit Model for CDRA}

A rectangular slot of width $=2 \mathrm{~mm}$ and length $=7 \mathrm{~mm}$ is used for the excitation of the CDRA. The length and width of the rectangular slots are adjusted so as to excite the CDRA for $H E M_{11}$ mode. The radiation resistance of the CDRA depends on the dimensions of the rectangular excitation slot [23].

The equivalent circuit model for the proposed CDRA along with the transmission line and a rectangular slot are shown in Figure 2. In the equivalent circuit model, the impedance transformer represents the $50 \Omega$ transmission line which excites the rectangular slot. Rectangular slot as well as cylindrical DRA is represented with the RLC resonator. The rectangular slot is responsible for installing the $H E M_{11}$ mode in the CDRA. In order to see the validity of the circuit model, the impedance and return loss plots are compared with the EM model. A decent agreement between the real part, imaginary part of the impedance and return loss of the circuit model and EM model is obvious from Figure 3. The optimized values for circuit model $\left(X_{1}, X_{2}, R_{\text {slot }}, L_{\text {slot }}, C_{\text {slot }}, R_{D R A}, C_{D R A}\right.$ and $\left.L_{D R A}\right)$ are listed in Table 1. The E and $\mathrm{H}$ fields of HEM 11 mode excited in the CDRA are shown in Figure 4. 


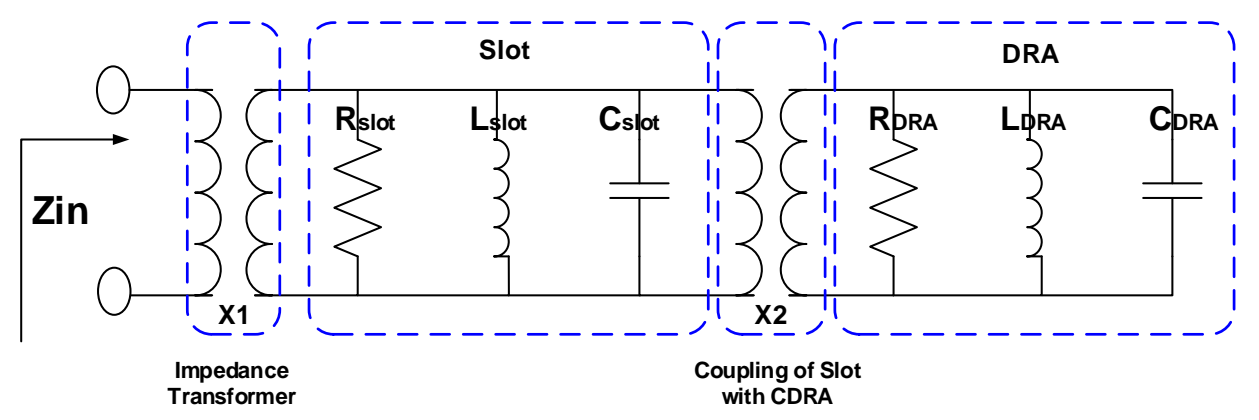

Figure 2. Equivalent circuit model of the complete antenna system.

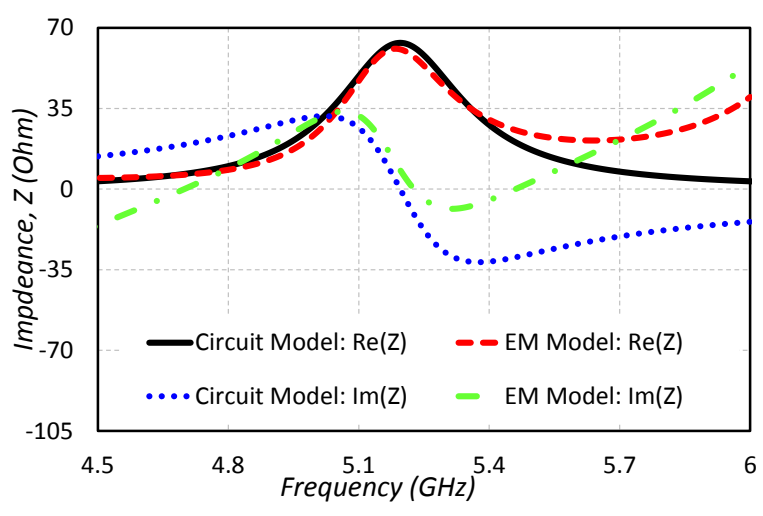

(a)

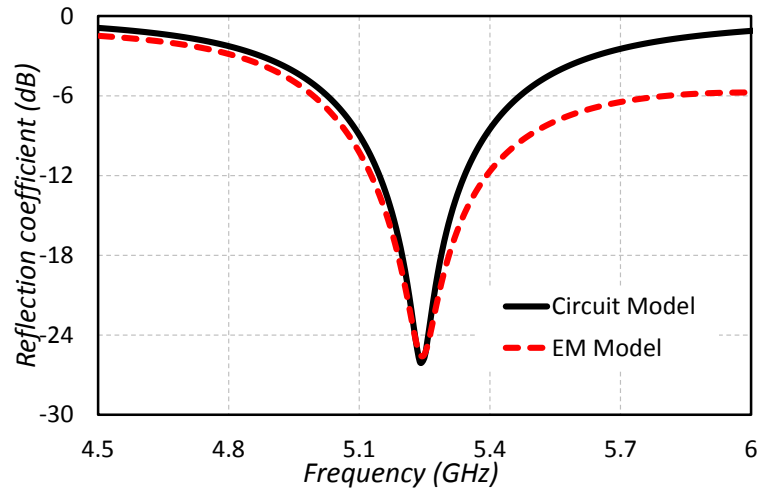

(b)

Figure 3. Comparison of the EM model and equivalent circuit model: (a) Impedance (b) Return loss.

Table 1. Equivalent circuit model components values.

\begin{tabular}{cccccc}
\hline Components & Values & Components & Values & Components & Values \\
\hline$R_{\text {slot }}$ & $250 \Omega$ & $R_{D R A}$ & $950 \Omega$ & $X_{1}$ & 0.50 \\
$C_{\text {slot }}$ & $0.981 \mathrm{pF}$ & $C_{D R A}$ & $1.915 \mathrm{pF}$ & $X_{2}$ & 1.382 \\
$L_{\text {slot }}$ & $0.67 \mathrm{nH}$ & $L_{D R A}$ & $0.793 \mathrm{nH}$ & & \\
\hline
\end{tabular}

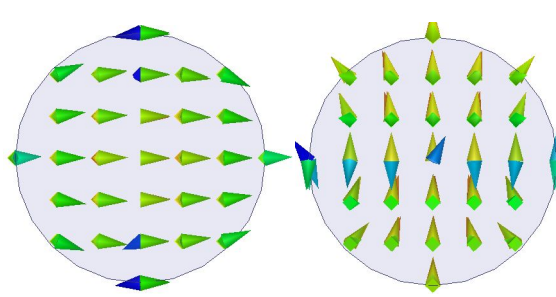

(a)

(b)

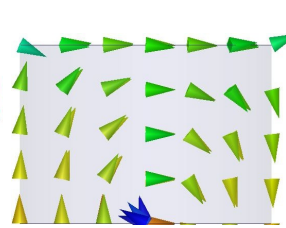

(c)

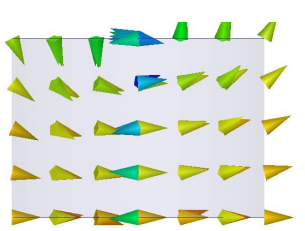

(d)

Figure 4. E-fields and H-fields distribution on DRA (a) Top view: E-fields (b) Top view: H-fields (c) Side view: E-fields (d) Side view: H-fields.

\subsection{DRA-Based Sensors Design}

\subsubsection{Type 1 Sensor}

The design of this type of sensor is shown in Figure 5a. A cylindrical dielectric resonator antenna is used as a sensing element in this work. A High-Frequency Structure Simulator (HFSS v13.0) is used for designing and optimization purposes. A cylindrical microwell with PDMS is installed in the center of the CDRA for the liquid under test (LUT). A microwell using PDMS values is extracted so as to 
give proper physical structure in simulations. A large number of simulations have been performed so as to find the optimized radius and height of the microwell basin, keeping in mind the unloaded quality factor of the dielectric resonator as well as the sensing capabilities. It is always desirable for an RF sensor to have a high quality factor so as to intelligently sense dielectric materials of a high dielectric constant value. The optimized microwell basin has the height of $6 \mathrm{~mm}$ and radius of $2 \mathrm{~mm}$. The reflection coefficient graphs for the type 1 sensor are given in Figure 6a. Four different types of liquid chemicals (Isopropyl, ethanol, methanol and water) are tested in the designed sensor and their results are shown in Figure 6a. It is clear from Figure 6a that the designed sensor behaves differently for all the liquid chemicals based on their dielectric constant value. The designed sensor is tested under six different conditions: one without PDMS microwell, second with no liquid in the basin, and then testing of four different liquid chemicals. The proposed CDRA has a resonance frequency of $5.25 \mathrm{GHz}$ in the absence of microwell for liquid chemical sensing. The resonance frequency of the CDRA shifts to $5.36 \mathrm{GHz}$ when the microwell is designed at the center of the CDRA. The right hand shift of the resonance frequency of the CDRA is due to the low permittivity of the air inside the microwell. When the microwell is filled with isopropyl, the resonance frequency shifts towards the lower frequency side and noted at $5.16 \mathrm{GHz}$. The frequency shift of the resonance frequency of CDRA is because of the higher dielectric constant of the isopropyl chemical than the CDRA and air [22]. The resonance frequency of the CDRA sensor is noted at $5.152 \mathrm{GHz}$, when the ethanol liquid chemicals is tested in the microwell. The resonance frequency shifts to $5.12 \mathrm{GHz}$ for methanol testing. In the case of testing water in the type 1 sensor, the resonance frequency shifts to $5.048 \mathrm{GHz}$. The large shift of the resonance frequency of type 1 sensor is because of the large dielectric constant value of the water. In this way, the proposed model can be utilized as a sensor using the varying behavior of the resonance frequencies for different liquid chemicals.

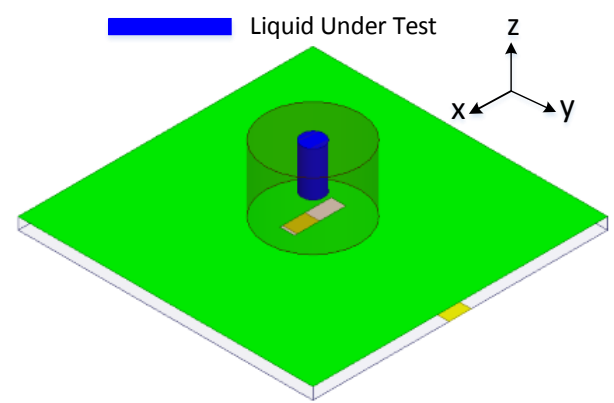

(a)

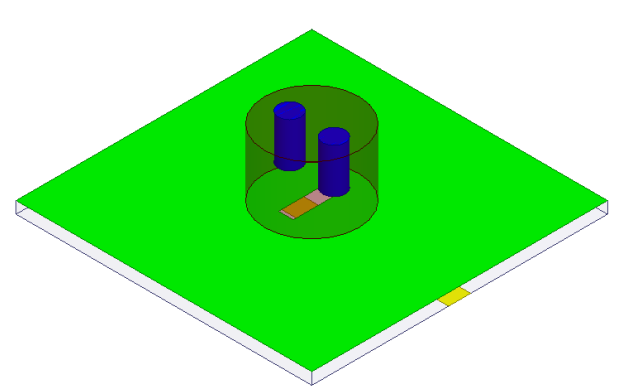

(b)

Figure 5. Types of DRA-based sensors (a) Type 1: One cylindrical microwell for liquid testing (LT) (b) Type 2: Two cylindrical microwells for LT.

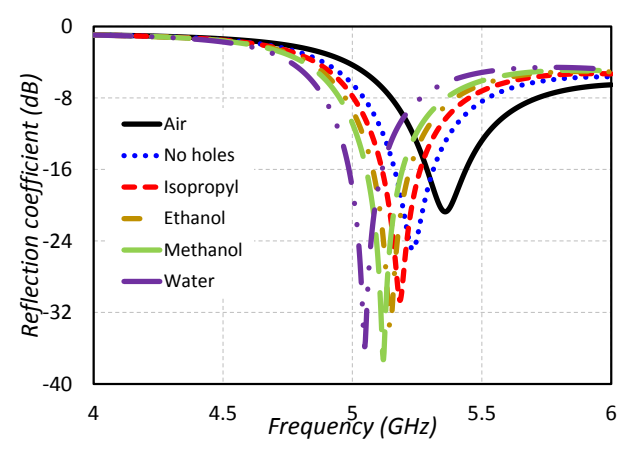

(a)

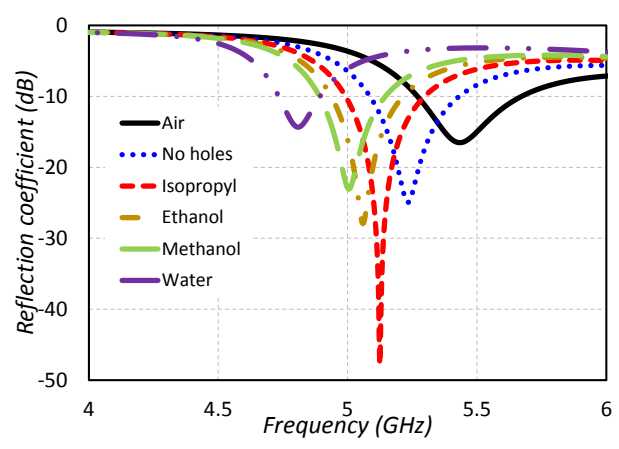

(b)

Figure 6. Reflection coefficient of the sensors for different liquid chemicals (a) Type 1 sensor (b) Type 2 sensor. 
Parametric analysis is done in order to discover the average sensitivity for the designed sensor. For this purpose, the loss tangent is kept at 0.4 which is the common value for ethanol at $3-5 \mathrm{GHz}$ and the permittivity is varied. The average sensitivity [24] is determined using the following relation.

$$
\text { Average Sensitivity }(\mathrm{S} 1)=\frac{\Delta F}{\Delta \epsilon}=8.75 \mathrm{MHz} / \epsilon_{r}
$$

The sensing capabilities and frequency shift against the varying radius and height of the microwell is investigated in Figure 7a,b. The following relations are used for determining the fractional change in frequency and the sensitivity [2].

$$
\begin{gathered}
\text { Fractional change in Frequency }(F)=\frac{f(m)-f(s)}{f(s)} \\
\text { Sensitivity }=\frac{\Delta F}{\Delta \epsilon^{\prime}}
\end{gathered}
$$

where $f(m)$ and $f(s)$ are the resonance frequencies with and without the liquid under test respectively.

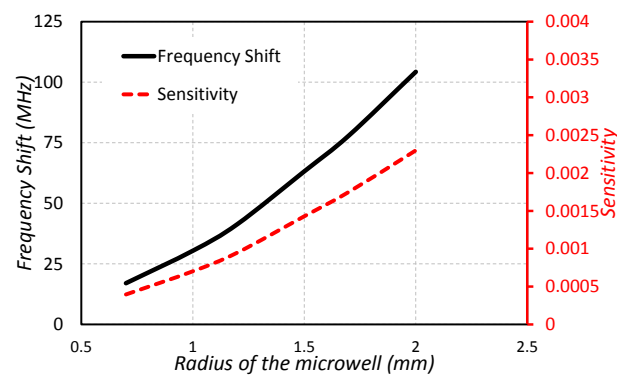

(a)

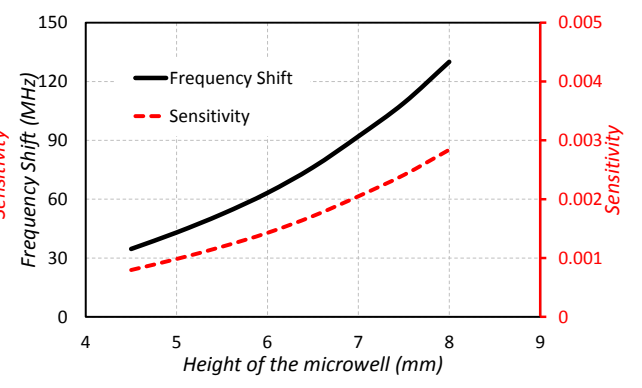

(b)

Figure 7. Type1: Frequency shift and sensitivity against (a) Radius of the microwell (b) Height of the microwell.

It is noted that the sensitivity and frequency shift values increase with the increase in radius and height of the microwell. The sensitivity and frequency shift of the sensor increase due to the large sample of the liquid chemical as well as more overlap area where the maximum fields exist.

\subsubsection{Type 2 Sensor}

This type of DRA-based sensor is shown in Figure 5b. The type 2 sensor is a modified form of the type 1 sensor. Two microwells of the same dimensions for liquid testing are designed in the type 2 sensor. The radius, height and position of the microwell are adjusted so that the sensor has high sensitivity, as well as high quality factor is ensured for sensing the high dielectric constant liquid chemicals. The sensor is tested for four different liquid chemicals and their results are shown in Figure $6 \mathrm{~b}$. The average sensitivity is computed as $10.28 \mathrm{MHz} / \epsilon_{r}$. The resonance frequency of the sensor is noted as $5.25 \mathrm{GHz}$ for no microwell in the CDRA. The resonance frequency shifts towards the higher frequency when the two microwells are designed in the CDRA. The resonance frequency is noted as $5.432 \mathrm{GHz}$ for no liquid under test but in the presence of two microwells. The resonance frequency is noted as $5.124 \mathrm{GHz}$ for isopropyl testing. The resonance frequency shifts towards the lower frequency side when the microwells are filled with ethanol chemicals. The resonance frequency in case of ethanol-filled microwells is noted as $5.056 \mathrm{GHz}$. The resonance frequency further shifts towards the lower frequency side when the methanol liquid is inserted in the microwells. The frequency for methanol-filled microwells is observed as $5.004 \mathrm{GHz}$. A large frequency shift towards the lower frequency side is obvious from Figure $6 \mathrm{~b}$, when the microwells are filled with water. The resonance frequency lies at $4.80 \mathrm{GHz}$ for water-filled microwells. Hence, it is proved that the proposed model can be used as a sensor to differentiate different liquids using the resonance frequency. The sensitivity and 
frequency shifts of the proposed sensor for varying radius and height of the microwells are investigated in Figure 8a,b.

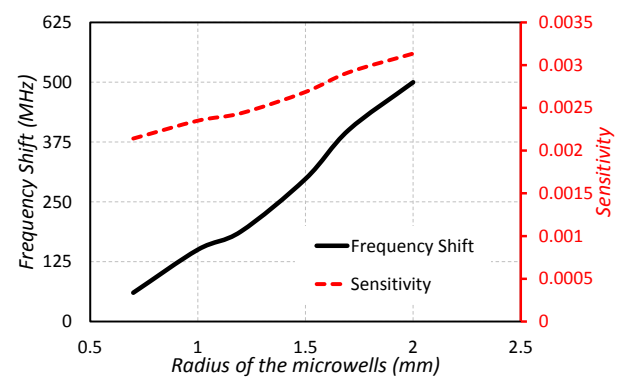

(a)

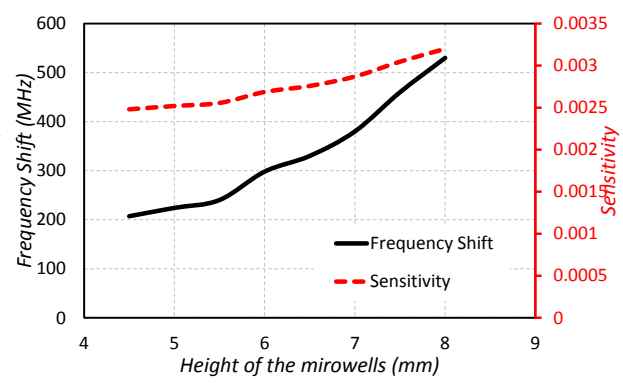

(b)

Figure 8. Type2: Frequency shift and sensitivity against (a) Radius of the microwells (b) Height of the microwells.

For measuring the proposed sensors, the reflection coefficient is measured after calibration of the vector network analyzer (VNA). First of all, the reflection coefficient of the sensors without any liquid (empty microwell) is measured by connecting the sensors one by one with VNA. After that, various liquid chemicals are injected into the microwells for chemical sensing. After each measurement, the PDMS microwell is cleaned by exerting air through a pressure syringe and reinstating the reference frequency originating from the empty PDMS microwell. Much consideration is required for the cleaning process and reinstating the reference frequency because contamination may cause a high degree of error in liquid chemical sensing processes. It is noted that all the liquid chemicals tested have different resonance frequencies. Figure 9 shows the measured responses of the proposed sensors. The sensors are fabricated and measured for water and isopropyl.

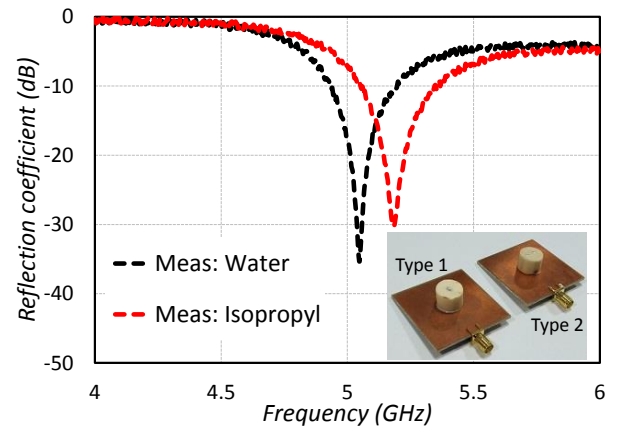

(a)

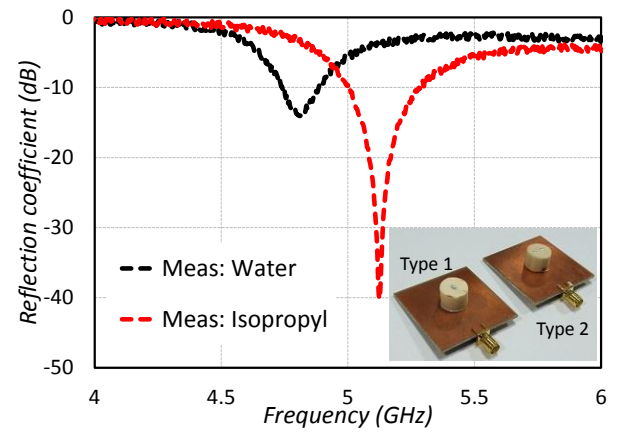

(b)

Figure 9. Measured results for CDRA sensor (a) Type 1 sensor (b) Type 2 sensor.

Table 2 summaries the performance of the two sensors. Table 3 shows the comparative analysis of the proposed sensors with existing RF sensors. It can be well-noted that the proposed sensors have low size, high frequency shift and high sensitivity compared to existing sensors.

Table 2. Comparison of the two types of sensors.

\begin{tabular}{ccccc}
\hline No. & Resonance Frequency & Average Sensitivity ${ }^{* 1}$ & $\boldsymbol{\Delta} \boldsymbol{F}^{* \mathbf{2}}$ & Sensitivity ${ }^{* 3}$ \\
\hline Type 1 Sensor & $5.25 \mathrm{GHz}$ & $8.75 \mathrm{MHz} / \epsilon$ & $202 \mathrm{MHz}$ & 0.0024 \\
Type 2 Sensor & $5.25 \mathrm{GHz}$ & $10.28 \mathrm{MHz} / \epsilon$ & $450 \mathrm{MHz}$ & 0.0031 \\
\hline${ }^{* 1}$ represents the average sensitivity obtained through relation of $\frac{\Delta F}{\Delta \epsilon} \cdot{ }^{* 2}$ represents the maximum frequency \\
shift among all the chemicals. ${ }^{* 3}$ is the sensitivity obtained through (5).
\end{tabular}


Table 3. Comparison with the already designed sensors.

\begin{tabular}{ccccccc}
\hline Ref. & Size $\left(\lambda_{\boldsymbol{g}}\right)$ & $f_{\mathbf{0}} \mathbf{( G H z )}$ & Technology & $\Delta \mathbf{F} \mathbf{( M H z})$ & Sensing & Sensitivity $\left(\mathbf{M H z} / \epsilon_{r}\right)$ \\
\hline$[8]$ & $0.5 \times 0.5$ & 4.4 & SIW & 500 & Single & $9.35-101 \mathrm{KHz} / \mathrm{RH}$ \\
{$[12]$} & NA & $4-5$ & SIW & $112.1,265.7$ & Dual & $17.5,25.7$ \\
{$[15]$} & $1.25 \times 1.25$ & 5 & SIW & 380 & Single & 69.07 \\
{$[16]$} & $0.21 \times 0.21$ & $2.02,3.34$ & Microstrip & 281,309 & Dual & $3.57,3.9$ \\
{$[17]$} & NA & 4.7 & SIW & 70 & Single & 12.73 \\
{$[18]$} & $0.43 \times 0.93$ & 5.85 & SIW & 38 & Single & 5.84 \\
{$[19]$} & $1.7 \times 1.98$ & 17 & SIW & 145 & Single & 26.36 \\
Type 1 Sensor & $\mathbf{0 . 5 2} \times \mathbf{0 . 5 2}$ & $\mathbf{5 . 2 5}$ & DRA & $\mathbf{2 0 2}$ & Single & $\mathbf{8 . 7 5}$ \\
Type 2 Sensor & $\mathbf{0 . 5 2} \times \mathbf{0 . 5 2}$ & $\mathbf{5 . 2 5}$ & DRA & $\mathbf{4 5 0}$ & Single & $\mathbf{1 0 . 2 8}$ \\
\hline
\end{tabular}

\section{Conclusions}

A minimal size cylindrical dielectric resonator antenna (CDRA) using radio frequency signals to identify different liquids is successfully designed, simulated, fabricated and measured in this paper. The rectangular slot has been used to excite the CDRA for $H E M_{11}$ mode at $5.25 \mathrm{GHz}$. Circuit model values (capacitance, inductance, resistance and transformer ratios) of the proposed CDRA are derived to show the true behaviour of the system. Two different types of sensors are successfully implemented using CDRA. Different resonating frequencies are noted for the different types of chemicals using the two CDRA-based sensors (Type 1 sensor: air $=5.36 \mathrm{GHz}$, isopropyl $=5.16 \mathrm{GHz}$, ethano $=5.152 \mathrm{GHz}$, methanol $=5.12 \mathrm{GHz}$ and water $=5.048 \mathrm{GHz}$, Type 2 sensor: air $=5.432 \mathrm{GHz}$, isopropyl $=5.124 \mathrm{GHz}$, ethanol $=5.056 \mathrm{GHz}$, methanol $=5.004 \mathrm{GHz}$ and water $=4.80 \mathrm{GHz}$ ). An important feature of this sensor is its high quality factor during all chemicals sensing. Also, the sensor can be used as antenna when required due to its high quality factor and good impedance matching at resonance frequency.

Author Contributions: A.I. and A.S. provided the idea, performed the experiments and managed the paper. O.A.S., Q.H.A., N.K.M. and B.M.L. assisted in the idea development and paper writing.

Funding: This work was supported by the Basic Science Research Program through the National Research Foundation of Korea (NRF) funded by the Ministry of Education (grant number: NRF-2017R1D1A1B03028350).

Conflicts of Interest: The authors declare no conflict of interest.

\section{References}

1. Wei, Z.; Huang, J.; Li, J.; Xu, G.; Ju, Z.; Liu, X.; Ni, X. A High-Sensitivity Microfluidic Sensor Based on a Substrate Integrated Waveguide Re-Entrant Cavity for Complex Permittivity Measurement of Liquids. Sensors 2018, 18, 4005. [CrossRef] [PubMed]

2. Yasin, A.; Rehman, F.U.; Naeem, U.; Khan, S.A.; Shafique, M.F. Top Loaded TM01 $\delta$ Mode Cylindrical Dielectric Resonator for Complex Permittivity Characterization of Liquids. Radioengineering 2016, 25, 715. [CrossRef]

3. Cook, B.S.; Cooper, J.R.; Kim, S.; Tentzeris, M.M. A novel inkjet-printed passive microfluidic RFID-based sensing platform. In Proceedings of the 2013 IEEE MTT-S International Microwave Symposium Digest (MTT), Seattle, WA, USA, 2-7 June 2013; pp. 1-3.

4. Cook, B.S.; Cooper, J.R.; Tentzeris, M.M. Multi-layer RF capacitors on flexible substrates utilizing inkjet printed dielectric polymers. IEEE Microw. Wirel. Compon. Lett. 2013, 23, 353-355. [CrossRef]

5. Hofmann, M.; Fischer, G.; Weigel, R.; Kissinger, D. Microwave-based noninvasive concentration measurements for biomedical applications. IEEE Trans. Microw. Theory Tech. 2013, 61, 2195-2204. [CrossRef]

6. Mullett, W.M.; Levsen, K.; Lubda, D.; Pawliszyn, J. Bio-compatible in-tube solid-phase microextraction capillary for the direct extraction and high-performance liquid chromatographic determination of drugs in human serum. J. Chromatogr. A 2002, 963, 325-334. [CrossRef]

7. Dahlgren, R.; Nieuwenhuyse, E.; Litton, G. Transparency tube provides reliable water-quality measurements. Calif. Agric. 2004, 58, 149-153. [CrossRef] 
8. El Matbouly, H.; Boubekeur, N.; Domingue, F. Passive microwave substrate integrated cavity resonator for humidity sensing. IEEE Trans. Microw. Theory Tech. 2015, 63, 4150-4156. [CrossRef]

9. Carlborg, C.F.; Gylfason, K.B.; Kaźmierczak, A.; Dortu, F.; Polo, M.B.; Catala, A.M.; Kresbach, G.M.; Sohlström, H.; Moh, T.; Vivien, L.; et al. A packaged optical slot-waveguide ring resonator sensor array for multiplex label-free assays in labs-on-chips. Lab Chip 2010, 10, 281-290. [CrossRef]

10. Barrios, C.A. Optical slot-waveguide based biochemical sensors. Sensors 2009, 9, 4751-4765. [CrossRef]

11. Patko, D.; Mártonfalvi, Z.; Kovacs, B.; Vonderviszt, F.; Kellermayer, M.; Horvath, R. Microfluidic channels laser-cut in thin double-sided tapes: Cost-effective biocompatible fluidics in minutes from design to final integration with optical biochips. Sens. Actuators B Chem. 2014, 196, 352-356. [CrossRef]

12. Ndoye, M.; El Matbouly, H.; Sama, Y.N.; Deslandes, D.; Domingue, F. Sensitivity evaluation of dielectric perturbed substrate integrated resonators for hydrogen detection. Sens. Actuators A Phys. 2016, 251, 198-206. [CrossRef]

13. Chuma, E.L.; Iano, Y.; Fontgalland, G.; Roger, L.L.B. Microwave Sensor for Liquid Dielectric Characterization Based on Metamaterial Complementary Split Ring Resonator. IEEE Sens. J. 2018, 18, 9978-9983. [CrossRef]

14. Salim, A.; Memon, M.U.; Lim, S. Simultaneous Detection of Two Chemicals Using a TE20-Mode Substrate-Integrated Waveguide Resonator. Sensors 2018, 18, 811. [CrossRef] [PubMed]

15. Memon, M.U.; Lim, S. Microfluidic High-Q Circular Substrate-Integrated Waveguide (SIW) Cavity for Radio Frequency (RF) Chemical Liquid Sensing. Sensors 2018, 18, 143. [CrossRef] [PubMed]

16. Jankovic, N.; Radonic, V. A Microwave Microfluidic Sensor Based on a Dual-Mode Resonator for Dual-Sensing Applications. Sensors 2017, 17, 2713. [CrossRef] [PubMed]

17. Seo, Y.; Memon, M.U.; Lim, S. Microfluidic eighth-mode substrate-integrated-waveguide antenna for compact ethanol chemical sensor application. IEEE Trans. Antennas Propag. 2016, 64, 3218-3222. [CrossRef]

18. Liu, C.; Tong, F. An SIW resonator sensor for liquid permittivity measurements at C band. IEEE Microw. Wirel. Compon. Lett. 2015, 25, 751-753.

19. Memon, M.U.; Lim, S. Microwave Chemical Sensor Using Substrate-Integrated-Waveguide Cavity. Sensors 2016, 16, 1829. [CrossRef]

20. Hassan, A.; Lee, K.; Bae, J.; Lee, C.H. An inkjet-printed microstrip patch sensor for liquid identification. Sens. Actuators A Phys. 2017, 268, 141-147. [CrossRef]

21. Balanis, C.A. Antenna Theory: Analysis E Design; John Willey \& Sons: New York, NY, USA, 1997.

22. Petosa, A. Dielectric Resonator Antenna Handbook; Artech House Publishers: Norwood, MA, USA, 2007.

23. Naeem, U.; Iqbal, A.; Shafique, M.F.; Bila, S. Efficient Design Methodology for a Complex DRA-SIW Filter-Antenna Subsystem. Int. J. Antennas Propag. 2017, 2017, 6401810. [CrossRef]

24. Salim, A.; Lim, S. TM02 Quarter-Mode Substrate-Integrated Waveguide Resonator for Dual Detection of Chemicals. Sensors 2018, 18, 1964. [CrossRef] [PubMed]

(C) 2019 by the authors. Licensee MDPI, Basel, Switzerland. This article is an open access article distributed under the terms and conditions of the Creative Commons Attribution (CC BY) license (http://creativecommons.org/licenses/by/4.0/). 